\title{
Dual Band Optical Window (DBW) for Use on an EO/IR Airbome Camera
}

\author{
Kwang-Woo Park ${ }^{1}$, Sang-Yeong Park ${ }^{2}$, Young-Soo Kim ${ }^{2}$, Ki-Ho Kim ${ }^{3}$, and Young-Soo Choi ${ }^{1}$ \\ ${ }^{1}$ Agency for Defense Development, P.O. Box 35, Daejon 305-152, Korea \\ ${ }^{2}$ ISR Research Institute, Samsung Thales Co., Ltd, Chang-li 304, Namsa-myun, Yongin 449-885, Korea \\ ${ }^{3}$ Korea Electro-Optics Co., Ltd, Samjung-dong, Ojung-gu, Bucheon 421-150, Korea
}

(Received January 31, 2012 : revised February 17, 2012 : accepted February 28, 2012)

\begin{abstract}
This paper presents a method to derive the theoretical requirements for the development of a $400 \mathrm{~mm}$ optical window that transmits dual-band wavelengths and had a stable structure. We also present design and fabrication results. Among the required specifications, the surface figure error was defined by the transmitted wavefront deformation (TWD), $\lambda / 15 \mathrm{rms}$ at $632.8 \mathrm{~nm}$. This value was derived by estimating the predicted performances with respect to five independent items that could cause system performance degradation and then calculating the required wavefront error (WFE) to satisfy the performance goals. We measured the image resolution at each performance level to trace and verify the requirements. The article also describes a design optimization process that could minimize the weight and volume of the optical window attached to the payload securing the FOV of the camera. In addition, we accurately measured the deformation that occurred in the series of fabrication steps including processing, coating, assembly, bonding and bolting, and investigated the effects by comparing them to the results of a simulation performed in advance to derive the predicted performance.
\end{abstract}

Keywords: Optical window, Airborne camera, WFE budget, ZnS

OCIS codes : (220.4610) Optical fabrication; (120.4820) Optical systems; (280.4788) Optical sensing and sensors; (030.5570) Roughness; (220.5450) Polishing

\section{INTRODUCTION}

An EO/IR optical system that was supposed to be mounted as a payload in an airborne system was designed to satisfy a certain set of requirements. Main requirements were outer dimensions defined to place the system in the allocated volume, resolving power that depended on both optical characteristics and image quality, and functional durability in the extreme environmental conditions. This system was comprised of various components including a yoke, a housing, a gimbal for stabilization, an EO/IR sensor and an optical window for dual band. The EO/IR sensor of the system was designed to provide high-resolution images by thermal IR at night and by visible (EO) in daytime, or by both simultaneously at far-off ranges[1-2]. It was mounted on a two-axis gimbal that provided stabilized imaging and precision pointing in both along the track and cross-track directions.

The optical window was positioned on the outer cover of the payload. The purpose of this optical window was to provide a clear aperture for the desired radiations (Visible and Infrared) while keeping the EO/IR sensor protected from the outer harsh environment. Thus, it was essential that it be able to bear any atmospheric changes such as temperature and pressure because this system was supposed to be operated at high altitude [3].

This paper described the methods for determining the theoretical requirements of the large $(413 \mathrm{~mm} \times 313 \mathrm{~mm} \times$ $15 \mathrm{~mm}$ ) multispectral window (transmits both visible and infrared) employed for the airborne camera's primary sensor system. The requirements derived were verified by measuring the image resolutions during the fabrication and evaluation stages. We measured every possible deformation at every step from fabrication to assembly (bonding, bolting) because the optical system was very sensitive to deformation in this system, and those were compared with the calculated data from the simulation performed previously to define the requirements.

\footnotetext{
*Corresponding author: hoya12234@gmail.com

Color versions of one or more of the figures in this paper are available online.
} 


\section{WINDOW REQUIREMENTS AND SPECIFICATIONS}

\subsection{Requirement and Specifications}

Detailed specifications for the final coated DBW were developed based on the operational requirements of the EO/IR camera system and the optical performances of the EO/IR sensor. To determine the requirements, a great deal of consideration was required because it was the first attempt to use a $\mathrm{ZnS}$ (Cleartran) window with diameter $400 \mathrm{~mm}$ for dual band in Korea. The primary requirements for the design and fabrication of the $\mathrm{ZnS}$ (Cleartran) window for dual band can be summarized as follows.

Just like the primary and secondary mirror features, which were optically very critical in the high-resolution reflective optics, the wavefront deformation of the DBW was also a very important parameter for achieving the high-resolution image quality. In this report, the surface figure error of the DBW was defined by transmitted wavefront deformation (TWD) as $\lambda 15 \mathrm{rms}$ at $632.8 \mathrm{~nm}$ across 11 -inch aperture. This was determined as manufacturing error value and derived through WFE allocation.

The required optical MTF value was provided by the system allocation process. System optical MTFs, including all the degradation factors listed below were $0.3(\mathrm{EO}) / 0.2(\mathrm{IR})$.

- Optics Manufacturing Error

- Design + Alignment Error

- Assembly Error

- Environmental Error (Gravity, Temperature etc.)

- Other (Interface, Unknown factors, etc.)

Nevertheless, the MTFs were not convenient parameters for performance analysis. Therefore, these allocated MTF values were translated into WFE degradation parameters. The rms WFE was the most convenient parameter to quantify the optical image quality degradation obtained through several types of analyses (thermal, mechanical and optical). The Shannon formula was used as a tool to obtain rms WFEs from the allocated MTF degradation values [4-5]. The Shannon formula is described in Eq. (1)

$$
K_{\text {MTF }}=\frac{M T F_{\text {final }}}{M T F_{\text {initial }}}=1-k \cdot W F E^{2} \cdot \frac{v_{\text {nyquist }}}{v_{\text {cut-off }}} \cdot\left(1-\frac{v_{\text {nyquist }}}{v_{\text {cut-off }}}\right)
$$

where the k-factor that is dependent on the type of aberration was determined based on previous experience. Fig. 1 shows a list of rms WFE item allocation for the DBW.

The Design + Alignment parameter was residual assembly/ alignment error on the window wavefront, mainly due to the assembly of optics with structure, according to the tolerances allocated for each component. The expected performances were derived from initial assembly and manufacturing errors such as follows : Wedge angle (6 arc-sec), Thickness $( \pm 0.3 \mathrm{~mm})$, Decenter $( \pm 0.5 \mathrm{~mm})$, Tilt $\left( \pm 0.5^{\circ}\right)$, Position $( \pm 0.3 \mathrm{~mm})$.

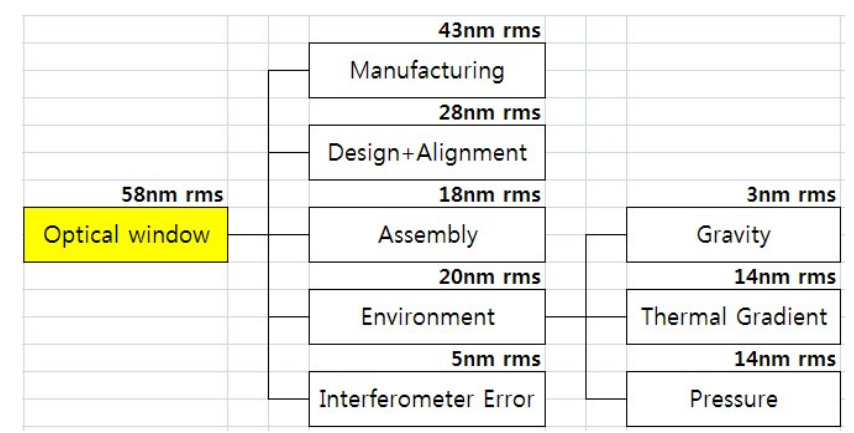

FIG. 1. List of WFE allocations for the DBW.

The performance predicting simulation was carried using the Code- $\mathrm{V}$ macro. We made a displacement error occurrence macro so that we could input values within the tolerances for the 5 items mentioned above. Within $\pm 2 \sigma(95.4 \%)$ ranges, which were derived by performing 1000 times in the macro, we obtained optimum prediction performance results for a given optical product by taking into account the various displacement errors that occur in mass production[6-9]. The Assembly parameter could be induced during assembly of the structure as well as during stress release after mounting. Expected performances for bonding effects were derived by the pilot test between two sheets of BK7 glasses of the same size as the $\mathrm{ZnS}$ window and frames. The deformation from bonding effects was $12.7 \mathrm{~nm}$ rms at each surface.

The Environment parameter represented the optical components, the mechanical interfaces and the structures. Analysis was performed for three considerations : Gravitational effect (induced by $1 \mathrm{G}$ gravity since the system was assembled and aligned on ground but would be used in the air. It concerned both the structure and the optics), thermal gradient effects (induced by the temperature differences between inner and outer surfaces at the optical window during the operation), and pressure difference effects(to investigate these effects, analysis was performed within the pressure range of $1 \mathrm{Kpa} \sim 10 \mathrm{Kpa})$. The predicted optical performances in the cases considered above were then analyzed using the Zernike polynomial decomposition method.

Eventually, Manufacturing error $(\lambda 15 \mathrm{rms}$ at $632.8 \mathrm{~nm})$ was determined as differences between total window budgets to satisfy the system MTF and the sum of optical allocation values (Design + Alignment, Assembly, Environment and Interferometer errors).

As shown in Fig. 1, the Manufacturing parameter was the largest among the optical budgets, so we should seriously consider putting more effort into the fabrication of this window.

\subsection{Designs}

The window should be large enough so it can secure the F.O.V of the sensor in order to satisfy the required image coverage requirement.

A forward motion compensation scheme was mechanically 
implemented to eliminate image smear due to the platform's forward motion [10]. The gimbal inside the payload was designed to rotate the F.O.V in pitch direction up to $\pm 15^{\circ}$ as shown in Fig. 2.

Since the outer gimbal that the window was mounted to did not include the driving mechanism, the window should be expanded further in pitch direction in order to secure the camera's field of view.

The design of the DBW was iterated 3 times, reflecting considerations of the operation concepts of the gimbal. Optimized window configuration and analysis results are shown in Table 1 below. The window thickness was selected based on a structural analysis using a simple edge-supported model of the window installation, and $15 \mathrm{~mm}$ thickness was selected for the DBW.

Initially, a window that has octagon shapes with $600 \mathrm{~mm}$ $\times 400 \mathrm{~mm}$ dimension was designed, but the concentrated stress that occurred at the edge area was shown in the 1st

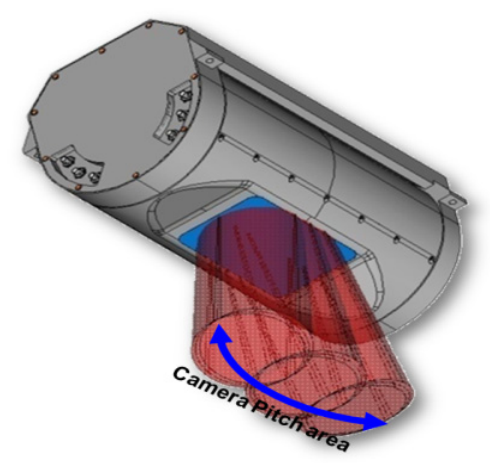

FIG. 2. Pitch movement of the EO/IR system (angle : $\pm 15^{\circ}$ ).
Model of TABLE 1 above. In order to reduce the stress concentration at the edge of the window, it has been modified from an octagonal shape to a rounded one. The revised window of $460 \mathrm{~mm} \times 350 \mathrm{~mm}$ shown in the 2 nd Model of TABLE 1 above had uniformly distributed stress and reduced volume and weight. The reduction rates of the volume and weight were $30 \%$ and $50 \%$, respectively.

Although both inner and outer gimbals could be driven in the roll direction during scanning, there was a possibility that their rotation angles did not match exactly. The roll angle errors for the inner and outer gimbals were estimated as from $\pm 2^{\circ}$ to $\pm 1^{\circ}$ by considering the system's dynamic characteristics. Therefore, another $\pm 1^{\circ}$ should be added to the maximum roll angle range. An optimized optical window that secures the camera's field of view was finally designed after considering this limitation in addition (3rd Model in TABLE 1). Size $413 \mathrm{~mm} \times 313 \mathrm{~mm}$ and edge radius of 150 $\mathrm{mm}$ were selected as the finally designed DBW configuration, which can be seen in Fig. 3. The 3rd Model is about 50\% the weight of the 1st Model. The 3rd Model shows light weight effects of about $50 \%$ compare to the 1st Model.

\section{FABRICATIONS}

\subsection{Measurements of the Optical Quality of CVD $\mathrm{ZnS}$}

$\mathrm{ZnS}$ (Cleartran) is a wide-band optical material and is one of the most widely used IR optical materials due to its good mechanical and thermal properties and theoretically excellent optical performances. It is known that bulk $\mathrm{ZnS}$ produced by CVD growth is very sensitive to the machining process[11-13]. Forward Scatter was selected as the inspection

TABLE 1. Optimization of the DBW

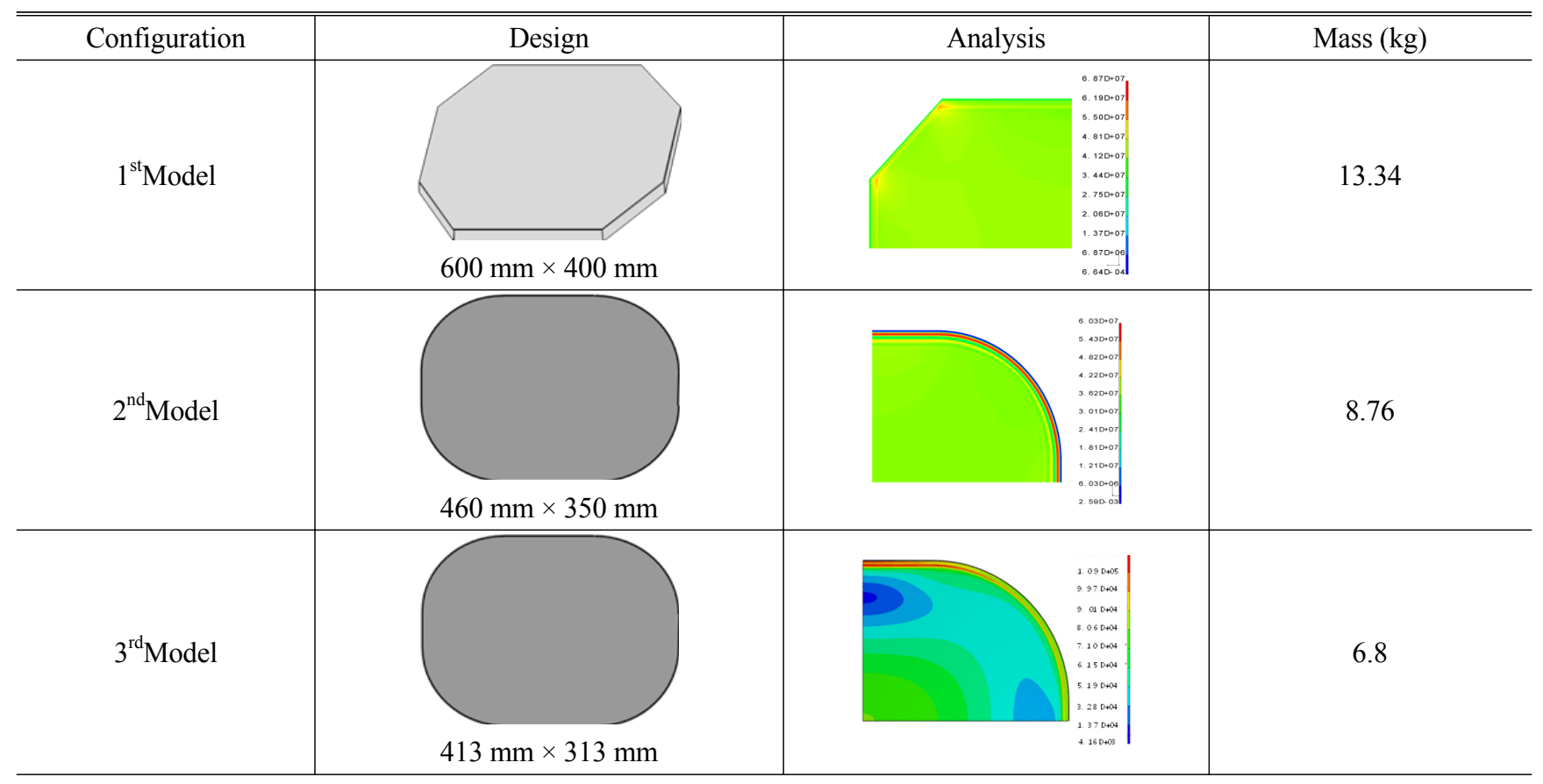




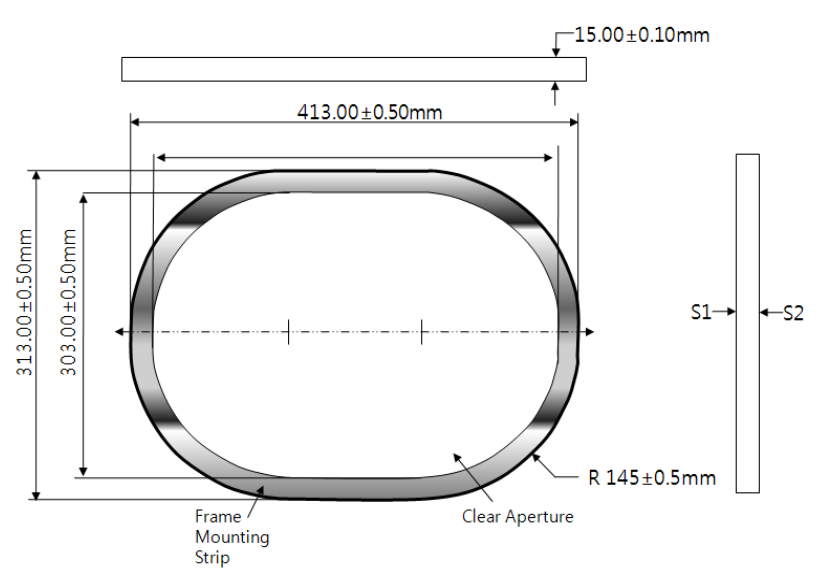

FIG. 3. Detailed view of the finally designed DBW.

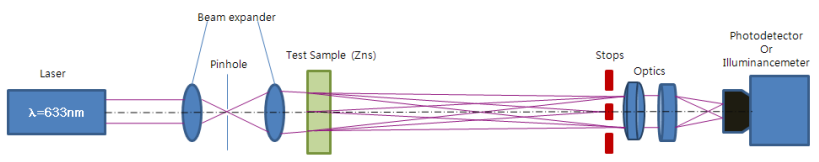

(a) Measure the scattered light, note intensity $I_{s}$

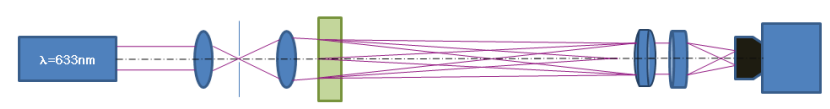

(b) Remove the aperture stop and measure laser output. Note intensity $I_{\Phi}$

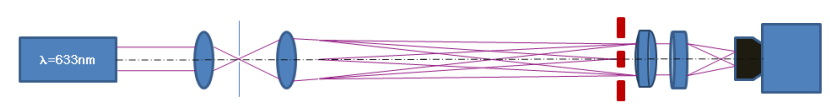

(c) Remove sample, put aperture stop, measure the offset or bias intensity $I_{a}$

FIG. 4. Methods of measuring the Forward Scatter of the ZnS.

method to control the degree of clarity of $\mathrm{ZnS}$ raw material. The procedure of the Forward Scatter method is as follows;

1. Put the $\mathrm{ZnS}$ sample just after the beam expander.

2. Measure the scattered light intensity IS. (Fig. 4(a))

3. Remove the stop and measure the laser output. Note the intensity signal Iф. (Fig. 4(b))

4. Remove the sample and put the stop in the detector unit. Repeat again step 2 and measure the offset or bias intensity Ia (Fig. 4(c))

5. Calculate the scattered light ratio as follows: $\mathrm{CS}=\{(\mathrm{IS}-\mathrm{Ia}) /(\mathrm{I} \phi)\} \mathrm{x}(1 / \mathrm{d})$ (Normalized on the sample thickness $\mathrm{d}(\mathrm{cm}))$

Forward scatter measurement results were below $5 \% / \mathrm{cm}$.

\subsection{Fabrication of the Optical Window}

We fabricated the DBW using the selected $\mathrm{ZnS}$ material. The critical requirements of the window were that TWD error should be less than $\lambda 15 \mathrm{rms}$, and the wedge also should be less than $6 \mathrm{arc} / \mathrm{sec}$ with surface quality (scratchscatter/dig) better than $60 / 40$. We first attempted a conventional polishing method with pitch tools and diamond slurry for final polishing.

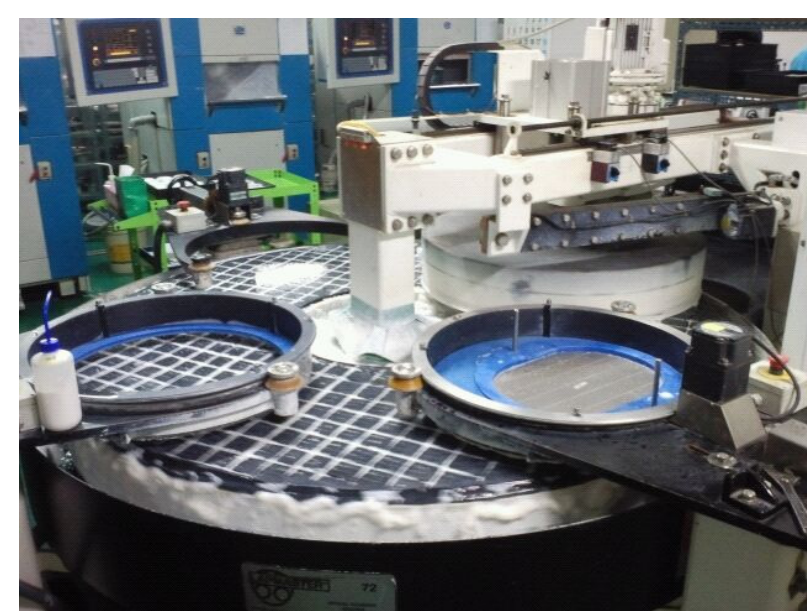

FIG. 5. DBW under polishing process on 72" LapMaster, with its own weight applied.

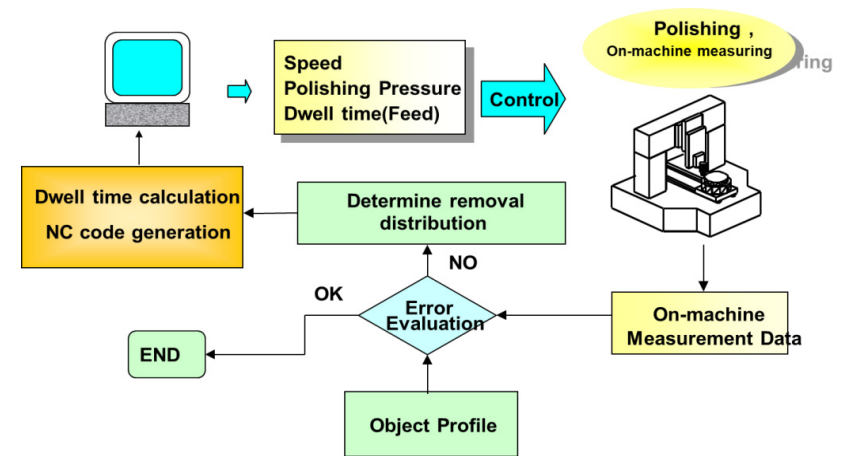

FIG. 6. Flow diagram of the correction polishing process.

Even though we obtained P-V value less than one lambda for every 11.8 " diameter clear aperture for each side of the window, the asymmetrical shape of the window made the TWD error worse than $\lambda / 3 \mathrm{rms}$. The fringe analysis result showed us that the TWD error is $1.65 \lambda$ RMS for the worst case for the conventionally polished window. In order to measure the quality exactly and to obtain feedback for the polishing process, we installed a new 12" interferometer.

As the window was quite thin (thickness was $15 \mathrm{~mm}$ ) relative to the size of the window (length was $413 \mathrm{~mm}$ on one side), normal jigging of the window might cause stress on the window. So, we used stress free polishing with 72 " Lapmaster equipment shown in Fig. 5. The result showed us better value $\lambda / 3 \mathrm{rms}$, but still didn't meet the requirement.

To secure TWD quality less than $\lambda / 15 \mathrm{rms}$, we performed conventional polishing followed by Lapmaster polishing. After those processes, we carried out correction polishing as described in the diagram below (Fig. 6). An in-situ 300 $\mathrm{mm}$ diameter Fizeau interferometer was developed additionally and used to monitor TWD error of the window in real time. Finally, the polished window showed us TWD less than $\lambda 15$ all over the whole clear aperture. Fig. 7 shows the configuration of the finally fabricated window. 


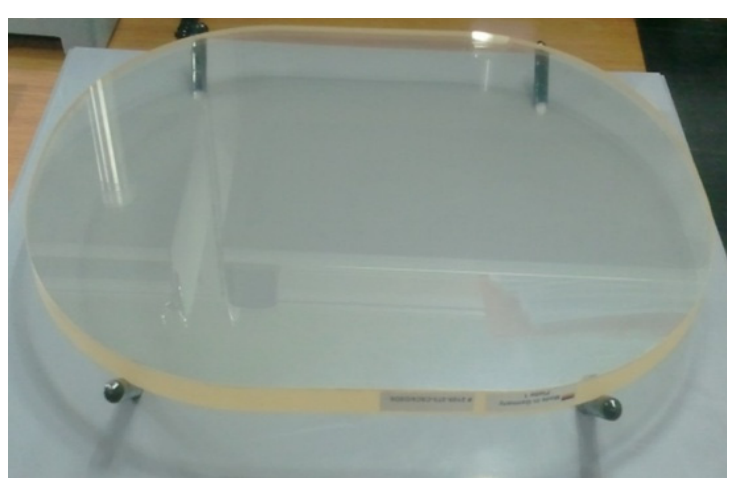

FIG. 7. Configuration of the finally fabricated DBW.

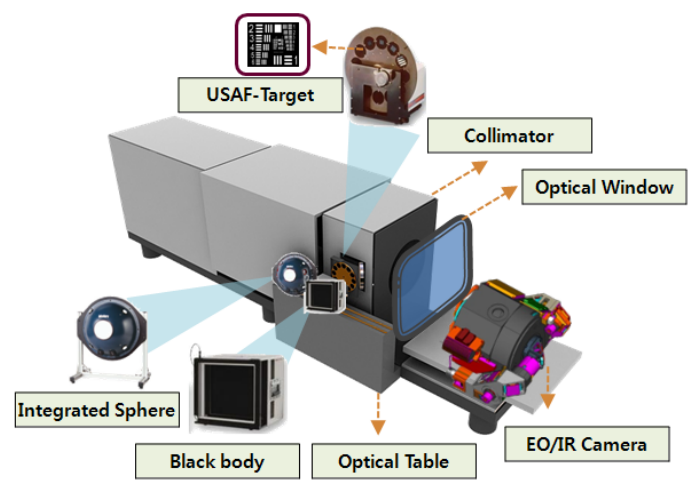

FIG. 8. Experimental set up for imaging resolution acquisition.

\subsection{Evaluation of the EO Camera Resolution by the Transmitted Wavefront Deformation (TWD)}

In this section, we explain the evaluation process of the EO camera resolution performance according to the transmitted WFE levels. Fig. 8 shows the equipment set up for image acquisition to measure the transmitted WFE levels.

The optical window was installed between the EO/IR camera and the collimator $(4 \mathrm{~m})$. The effect of imaging resolution according to the transmitted wavefront deformation (TWD) levels was confirmed by comparing the image before and after this window installation.

Table 2 shows the image performance for the DBW's TWD levels from $\lambda / 3 \mathrm{rms}$ to $\lambda / 8 \mathrm{rms}$. The required resolving power of the EO camera with the optical window was $4 \mathrm{G} 3 \mathrm{E} \sim 4 \mathrm{E}$ in the USAF 1951 chart. The measured resolution of the EO camera with window that was fabricated with TWD $\lambda$ $/ 8 \mathrm{rms}$ was $4 \mathrm{G1E}$ (4Group 1Element). These results showed about a $2 \sim 3 \mathrm{E}$ (Element) drop compared with the required resolution.

Table 3 shows the resolving power, which is $4 \mathrm{G} 4 \mathrm{E}$ in the USAF-1951 chart over any aperture at transmitted WFE $\frac{\lambda}{15}$ rms. (A) and (B) are window dimensions shown by rotating pitch direction $\left( \pm 15^{\circ}\right)$. These results were sufficient to satisfy the requirements of this system, and indicated that the initial conditions for transmitted WFE were set up correctly. This method can be used efficiently to define
TABLE 2. EO camera resolution according to the DBW's TWD levels (the fabricated DBW did not include a coating)

\begin{tabular}{|c|c|c|}
\hline Without the DBW & TWD $\frac{\lambda}{15} \mathrm{rms}$ & TWD $\frac{\lambda}{15} \mathrm{rms}$ \\
\hline 4 in 5 & $=\frac{F}{10}$ & \\
\hline in & 8111 & \\
\hline + $=111$ & aII & \\
\hline $4=111$ & aili & \\
\hline $4 \mathrm{G} 5 \mathrm{E}$ & Resolving (x) & $4 \mathrm{G} 1 \mathrm{E}$ \\
\hline
\end{tabular}

TABLE 3. Image resolution test results of the EO camera (@ TWD rms) (the fabricated DBW did not include a coating)

\begin{tabular}{|c|c|c|}
\hline Without the DBW & TWD $\frac{\lambda}{15} \mathrm{rms}(\mathrm{A})$ & TWD $\frac{\lambda}{15} \mathrm{rms}(\mathrm{B})$ \\
\hline$=\dot{1}=$ & 4 & \\
\hline 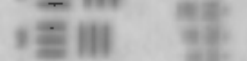 & ili & $s=i l l$ \\
\hline$t=111$ & 41 & $\varepsilon=5$ \\
\hline$t_{1 \mathrm{E}}$ & & 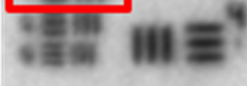 \\
\hline 4G5E & 4G4E & 4G4E \\
\hline
\end{tabular}

the surface form error levels for the development of large aperture optical windows.

\section{MEASUREMENT}

We measured all possible deformations at every step from fabrication to assembly (bonding, bolting), and the results were compared with simulation data which were previously performed to define the requirements.

Table 4 summarizes the equipment configuration for testing of the TWD, and shows fringe data at every step. The TWD for the manufacturing (including a coating) and assembly (including an alignment) were $39.2 \mathrm{~nm} \mathrm{rms}$ and $44.3 \mathrm{~nm}$ rms, respectively. These results showed that the series of procedures from fabrication to assembly could be performed well compared with the expected data. The predicted simulation performance results for the fabrication (including a coating) and assembly (including an alignment and an interferometer error) were $43 \mathrm{~nm}$ rms and $54.6 \mathrm{~nm}$ rms (refer to Fig. 1).

The gasket was bolted at the edge of the DBW, which bonded with the outer cover. 3 step measurements were used to obtain the image resolution influence due to the window with bolted gasket. The first step was to measure the assembled EO/IR camera with only the gimbal (without the DBW). In the second step, the outer cover with the bonded window was added to this camera. The final step was to include the bolted gasket additionally. Table 5 shows this step and those image resolutions from the USAF-1951 
TABLE 4. WFE measurement result at each step of the process

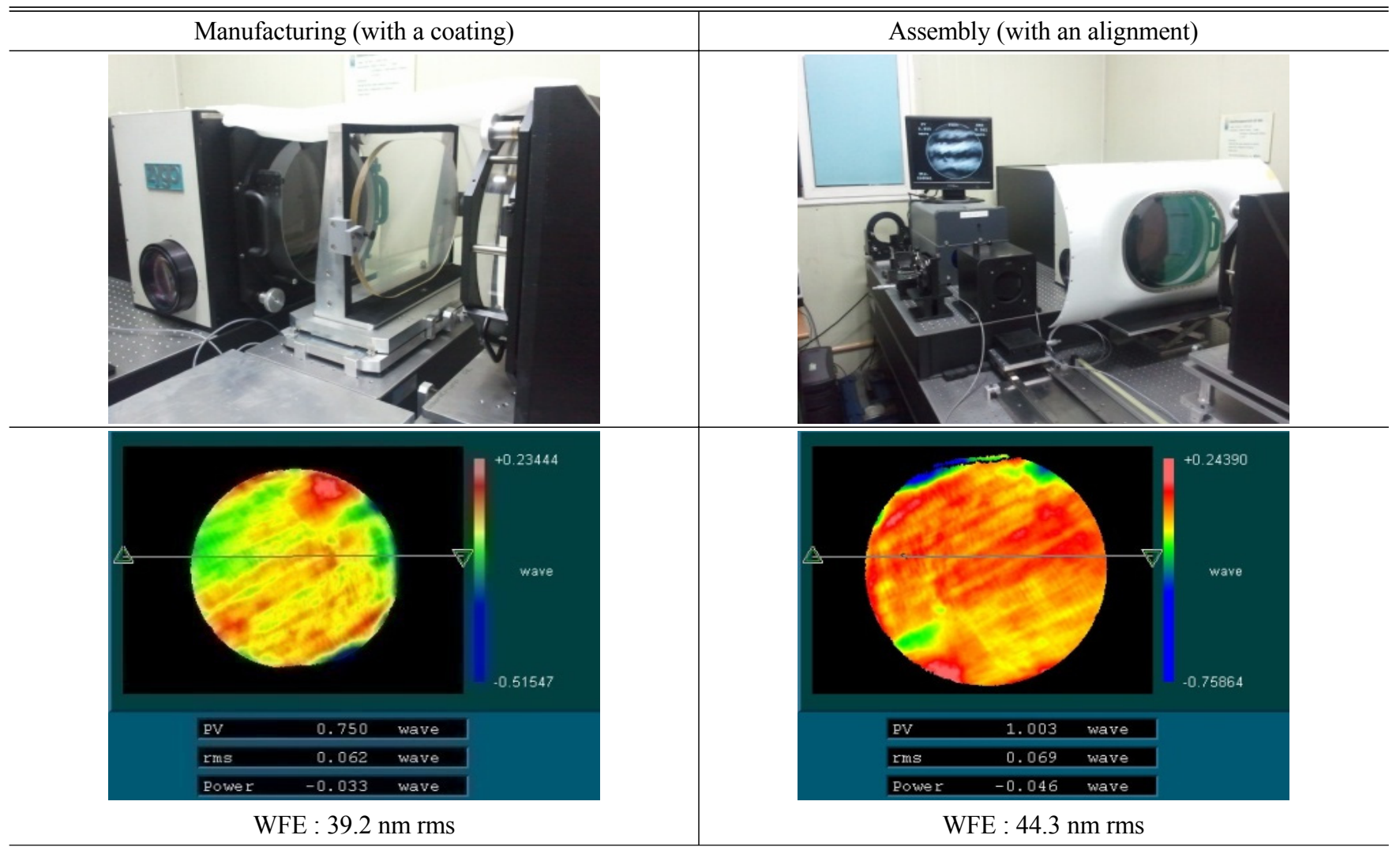

TABLE 5. Resolving power at every step from bonding to bolting. (in this experimens we considered only the assembly(bonding, bolting) work influence without environment effects such as temperature and pressure changes.)

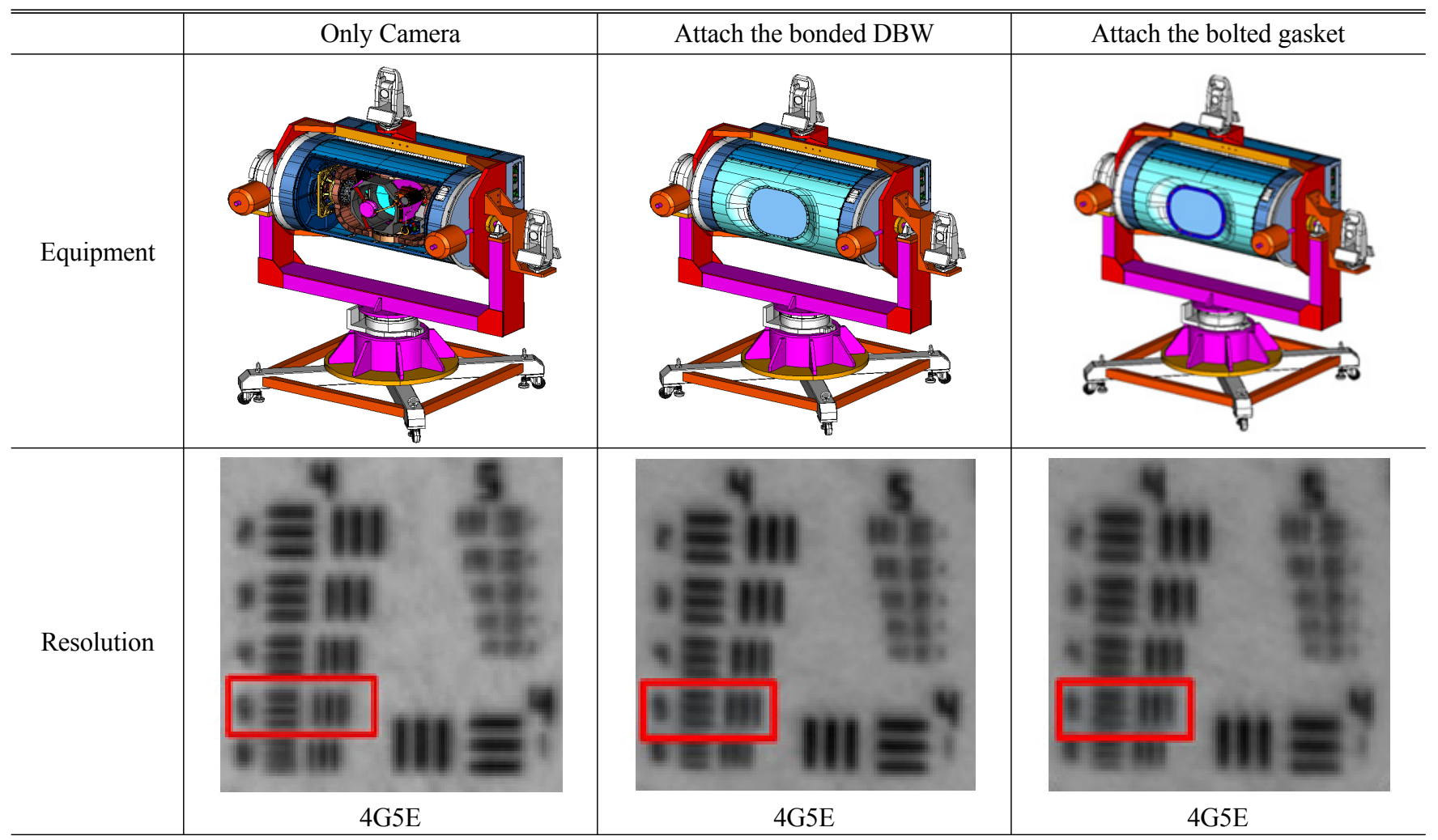


chart. Image resolutions before and after installation of the optical window were 4G5E in USAF-target, which means that there was no image degradation caused by the DBW.

\section{CONCLUSION}

This article presents a method that could be used to derive the theoretical requirements for the development of a $400 \mathrm{~mm}$ optical window with a stable structure. This article also shows the design and fabrication result. A light optical window was designed to secure the image for the camera and to ensure mechanical stability. The optical window was fabricated with the precision derived from the requirements (Transmitted Deformation Wavefront, $\frac{\lambda}{15} \mathrm{rms}$ ). The image resolution was verified in each step of the processing ( $\left.\frac{\lambda}{15} \mathrm{rms}, \frac{\lambda}{15} \mathrm{rms}, \frac{\lambda}{15} \mathrm{rms}\right)$. The image quality was not reduced if the precision reached $\frac{\lambda}{15} \mathrm{rms}$, showing that the detailed requirements were properly satisfied.

Deformation that occurred in the series of fabrication steps, including processing, coating, assembly and bonding, was accurately measured and compared with the result of the simulation that had been performed to derive the predicted performance. The measured results were $39.2 \mathrm{~nm}$ $\frac{\lambda}{15} \mathrm{rms}, 44.3 \mathrm{~nm}$ rms for manufacturing and assembly, which was much better than the predicted simulation performance result for assembly, $54.6 \mathrm{~nm}$ rms. Finally, we examined the effect on image resolution of bolting when the optical window was attatched to the outside of the payload. The results were 4G5E before the optical window attachment, 4G5E after the optical window attachment and 4G5E after the gasket bolting to the optical window rim.

These results showed that the series of steps from the derivation of the perfomance requirements of the optical window to the fabrication and assembly could be performed well without reducing the target resolution of the system (4G5E). This method is expected to be used efficiently for the further development of large-aperture optical windows.

\section{REFERENCES}

1. J. H. Lee, Y.-S. Jung, S.-Y. Ryoo, Y.-J. Kim, B.-U. Park, H.-J. Kim, S.-K. Youn, K.-W. Park, and H. B. Lee, "Imaging performance analysis of an EO/IR dual band airborne camera," J. Opt. Soc. Korea 15, 174-181 (2011).

2. J.-Y. Han, S. Marchuk, H. Kim, C.-W. Kim, and K.-W. Park, "Imaging EO/IR optical system for long range oblique photography" Proc. SPIE 8020, 802009 (2011).

3. C. J. Pruszyski, W. M. Ford, J. E. Rudisill, W. R. Stark Jr., and G. R. Anderson II, "Design, fabrication and testing of large airborne ZnSe windows," Proc. SPIE 2536, 366-375 (1995).

4. R. Shannon, The Art and Science of Optical Design (Cambridge University Press, Cambridge, UK, 1997), Chapter 4.

5. C. Olson, "Lens performance budgeting using the Hopkins ratio," (http://www.osa-opn.org/Content/ViewFile.aspx?id=10945).

6. Optical Research Associates, Code- $\mathrm{V}$ Reference Manuals ver, 9.3 (2003).

7. W. J. Smith, Modern Lens Design (McGraw-Hill, USA, 2005), Chapter 23.

8. D. Deshmaty-Manesh and G. Y. Haig, "Lens tolerancing by desk-top computer," Appl. Opt. 25, 1268-1270 (1986).

9. L. Gilles, L. Wang, and B. Ellerbroek, "Wavefront error budget development for the thirty meter telescope laser guide star adaptive optics system," Proc SPIE 7015, 701520 (2008).

10. A. G. Lareau, "Electro-optical imaging array with motion compensation," Proc. SPIE 2023, 65-79 (1993).

11. J. McCloy, "International development of chemical vapor deposited zinc sulfide" Proc. SPIE 6545, 654503 (2007).

12. C. H. Hargraves Jr. and J. M. Martin, "IR sensor and window system issues," Proc. SPIE 1760, 329-337 (1992).

13. http://www.dom.com/assets/attachments/business/gt/infrared materials/cleartran/tds/cleartran.pdf 